einfallenden Atome:

$$
\beta=N_{+} / N_{0} .
$$

$\beta$ wurde bisher noch für keine Kombination - einfallende Atome/glühende Oberfläche - absolut bestimmt.

Wir haben eine solche absolute Messung von $\beta$, vorerst für die Ionisation von Kalium an polykristallinen Wolframund Platin-Oberflächen, durchgeführt. Dabei wurde die Anzahl $N_{0}$ der aus einem Atomstrahl einfallenden Atome mit Hilfe einer in die Höchstvakuumapparatur eingebauten Quarz-Mikrowaage bestimmt; die Empfindlichkeit von $10^{-8} \mathrm{~g} /$ Ausschlagseinheit dieser auf dem Torsionsprinzip beruhenden Waage ${ }^{1}$, $\left.{ }^{2}\right)$ reicht zur Wägung selbst monoatomarer Schichten aus. Das verwendete Alkalimetall wurde durch mehrfache langsame Destillation in Hochvakuum, die Oberfläche der zur Ionisiernng verwendeten Metallfolien durch sorgfältiges Glühen im Höchstvakuum gereinigt; Adsorptionsschichten konnten sich auf den Folienoberflächen wegen des geringen Restgasdruckes von $<1 \cdot 10^{-9}$ Torr während der Dauer einer Meßreihe nicht bilden.

Der Strom der von der glühenden Metalloberfläche verdampfenden Ionen zeigt in einem Temperaturbereich (im vorliegenden Fall zwischen 1100 und $1200^{\circ} \mathrm{K}$ liegend) ein Maximum. Diesem Maximum des Kaliumionenstromes entspricht nach unseren Messungen eine Ionisierungsausbeute von $\beta=0,97 \pm 0,04$ bei Wolfram und von $\beta=0,99 \pm 0,03$ bei Platin. Kaliumatome werden also an sauberen polykristallinen Wolfram- und Platin-Oberflächen bei dieser Temperatur annähernd $100 \%$ ig ionisiert.

Der Deutschen Forschungsgemeinschaft danken wir für die Unterstützung dieser Untersuchungen durch Bereitstellung von Geräten.

Physikalisches Institut dev Technischen Hochschule für Bergbau und Hüitenwesen, Clausthal

Herbert Mayer und Walter Schroen

Eingegangen am 14. März 1962

1) Mayer, H., u. W. Schroen: Vortrag Dtsch. Phys. Tag. Wiesbaden 1960. - MAyer, H., W. Schroen u. D. StÜnkel. 1960 Seventh National Symposium on Vaccum Technology Transactions, S. 279. Oxford: Pergamon Press 1961. - . 2) NTedermayer, R., u. W. SChroen: Vak.-Techn. 11 (1962) (im Druck).

\section{Ein neues Sternendiagramm}

Die Bestätigung des Einflusses von Gravitationsfeldern auf die Verschiebung der Spektrallinien durch die Versuche über den Mößbauer-Effekt lassen es interessant erscheinen, die Dichte des Gravitationsfeldes an der Oberfläche verschiedener Sterne zu berechnen. Wir sind dabei so vorgegangen, daß wir in Analogie zur Maxwellschen Energiedichte in einem elektrischen Feld $E=(\varepsilon / 8 \pi) \mathbb{S}^{2}$ die Dichte der Gravitationsfeldenergie in der Form angesetzt haben $E_{g}=F_{g}^{2} /(8 \pi G)$, wobei $G$ die Gravitationskonstante ist, $F_{g}=G M / R^{2}$ die Gravitationsfeldstärke, $R$ ist Radius, $M$ die Masse des Sternes. Die "Gravitationsfelddichte" wird danach $\varrho_{g}=E_{g} / c^{2}, c$ Lichtgeschwindigkeit. $E_{g}$ hat die Dimension Energie/Volumen, daher hat $\varrho_{g}$ die Dimension Masse/Volumen. Das spezifische Volumen des Gravitationsfeldes ist $V_{g}=1 / \varrho_{g}$.

Wir haben die Werte $V_{g}$ an der Oberfläche von 400 Sternen berechnet und $\log V_{g}$ (als Ordinate) über der Oberflächentemperatur $T$ (als Abszisse) anfgetragen. Für die Hälfte der Sterne

Tabelle

\begin{tabular}{c|c|c|c|c|c|c|c|c|c}
\hline & $\log \alpha$ & $a$ & $b$ & $c$ & & $\log \alpha$ & $a$ & $b$ & $c$ \\
\hline 1 & $-5,66$ & 7,36 & $107 \cdot 10^{-8}$ & 20500 & 8 & $-5,88$ & 7,36 & $115 \cdot 10^{-8}$ & 17700 \\
2 & $-5,67$ & 7,38 & $115 \cdot 10^{-8}$ & 20500 & 9 & $-5,93$ & 7,54 & $170 \cdot 10^{-8}$ & 17500 \\
3 & $-5,74$ & 9,67 & $537 \cdot 10^{-9}$ & 37000 & 10 & $-5,97$ & 7,69 & $75 \cdot 10^{-8}$ & 21000 \\
4 & $-5,77$ & 7,84 & $692 \cdot 10^{-9}$ & 27000 & 11 & $-5,98$ & 8,60 & $120 \cdot 10^{-8}$ & 21000 \\
5 & $-5,78$ & 7,50 & $500 \cdot 10^{-9}$ & 32000 & 12 & $-6,01$ & 7,68 & $224 \cdot 10^{-8}$ & 12300 \\
6 & $-5,80$ & 8,70 & $742 \cdot 10^{-9}$ & 32000 & 13 & $-6,02$ & 9,14 & $324 \cdot 10^{-8}$ & 50000 \\
7 & $-5,83$ & 7,14 & $10^{-6}$ & 17500 & 14 & $-6,09$ & 9,28 & $339 \cdot 10^{-9}$ & 50000
\end{tabular}

liegt $\log V_{g}$ zwischen 6 und 8 . Rechts im Diagramm liegen die $A$-, $B$ - und $O$-Sterne, links oben die Giganten, links unten die roten $Z$ werge. Die ,Masse des Gravitationsfeldes" eines Sternes ergibt sich $2 \mathrm{n} \mu=\int_{R}^{\infty} \varrho_{g} 4 \pi R^{2} d R=G M^{2} /\left\{2 R c^{2}\right)$.

Wir haben insbesondere Sterne mit konstantem Verhältnis $\alpha=\mu / M=G M /\left(2 R c^{2}\right)$ ausgewählt. Für konstantes $\alpha$ gilt an- genähert die Gleichung

$$
\log V_{g}=a \pm b(c-T)^{\frac{3}{2}}
$$

Hierbei steht das Zeichen + für die Giganten, - für die Zwerge. Die Gleichung stellt V-förmige Kurven dar, die für $T=c$ eine Spitze haben und sich von da nach links öffnen. Die Tabelle zeigt die Zahlenwerte.

Von den untersuchten Sternen gehören zu Nr. 1: V 478 Cygn. A; V 470 Cygn. B; SX Aurigae A; i Boot. A. - 2: V 478 Cygn. B; $\mu$ Scorp. B; TZ Cancer A. - 3: UW Canis maj. A; V 470 Cygn. A; WW Cygn. A; $\mu$ Scorp. A. $-4:$ V 444 Cygn. A; V Pupp. B; UC Cor. Bor. A; $\alpha$ Gem. B; WZ Oph. A. 5: CC Cass. B; AG Pers. A; TV Cass. A; U Ceph. A; TX Urs. maj. B. - 6: AO Cass. A; VW Ceph. A; W 470 Cygn. A CM Lac. B; TT Aur. B; $\beta$ Pers. A. - 7: $\sigma$ Aquil. B; U Oph. B; $\alpha$ Leon.; AR Aurig. A; RX Gem. A. - 8: CV Vel. B; $\delta$ Ori. B; AR Aur. B; RX Triang. A; DO Herc. B. - 9: Z Vulp. A; $\beta$ Ori. B; TX Herc. B, Barnarda. - 10: Sonne. - 11: HD 1936; UV Peg. A; RY Pers. A; $\beta$ Aur. B; Y Pisc. A; RS Can. Ven. A. -12 : $\zeta$ Aur. B; RX Herc. A; $\lambda$ Oph. A; AH Virg. A; XY Gem. B. - 13: RY Sculpt. A; $\alpha$ Gem. C; RS Ceph. A. 14: RY Sculpt. B; VSO 2 Oph. A; UZ Pupp. B; W Urs. min. B; ER Ori. A.

USSR, Moskau, Serebrjanitschesky per., 9, 60.

Eingegangen am 23. Januar 1962

\section{N.P. SUWOROFF}

\section{Quantitative Messungen des Nebelniederschlages} im Walde von Fray-Jorge an der nordchilenischen Küste

Der Wald von Fray-Jorge $\left(30^{\circ} 30^{\prime}\right.$ südl. Br.) an der nordchilenischen Küste der Provinz Coquimbo ist von Botanikern und Okologen wiederholt beschrieben worden $\left.\left.{ }^{1}\right),{ }^{2}\right),{ }^{3}$ ). Er liegt mit seinem Schwerpunkt in $500 \mathrm{~m}$ Höhe über $\mathrm{NN}$ am Westhang eines Kordillerenzuges in 4 bis $5 \mathrm{~km}$ Entfernung von

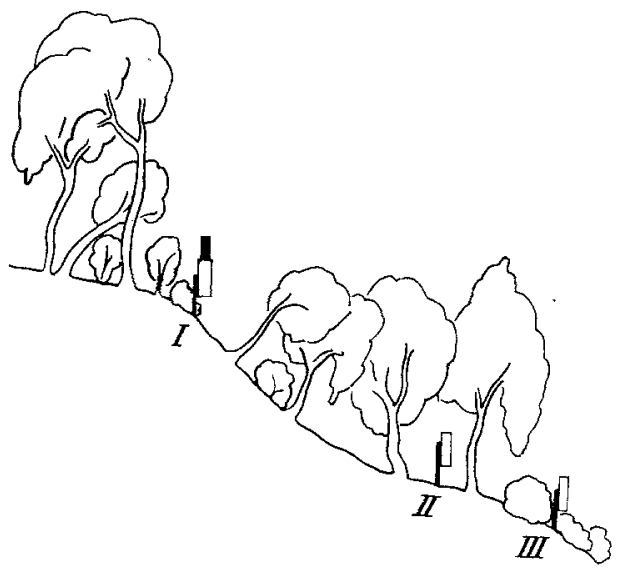

Fig. 1. Halbschematische Darstellung der Aufstellung der MeBinstrumente. I Nebelfärnger nach GRUNow, II Pluviometer zum Auffangen des aus der Baumkrone abtropfenden Wassers,
III Kontroll-Pluviometer

der Küste. Aus den Daten der nächstgelegenen meteorologischen Beobachtungsstationen ${ }^{4}$ ) läBt sich interpolieren, daß die durchschnittliche jährliche Regenmenge $150 \mathrm{~mm}$ nicht überschreitet. Es kommen aber auch extrem feuchte (300 mm) und völlig niederschlagsfreie Jahre vor. Der aride Charakter der Zone wird schließlich dadurch erhöht, daß sich die Regenfälle auf die Monate Mai bis August beschränken. Der Wald selber trägt floristisch deutlich die Züge des valdivianischen Regenwaldes, eines Gebietes, in welchem 1000 bis $2500 \mathrm{~mm}$ Niederschlagshöhe gemessen werden. Das demnach für unsern Wald vorhandene Regendefizit muß von den häufigen Nebeln gedeckt werden. Da bislang nur einige Beobachtungen über die Nebelhäufigkeit vorliegen ${ }^{1}$ ), wurden von uns ein Nebelfänger des Hohenpeissenberger Modells nach GRuNow ${ }^{5}$ ) sowie zwei gewöhnliche Pluviometer im Walde aufgestellt. Einen Eindruck über die Position der Instrumente vermittelt Fig. 1. Der eigentliche Nebelfänger (Instrument I) steht frei vor dem Waldrand, dem sehr beständigen Südwestwind direkt ausgesetzt. Instrument II ist ein gewöhnlicher Regenmesser, der unter der Krone eines etwa $5 \mathrm{~m}$ hohen Exemplars von Myrceugenia correifolia $8 \mathrm{~m}$ vom Waldrand entfernt installiert 